\title{
A Clinical and Aetiological Study of Hyponatremia in Patients Admitted to Medical Intensive Care Unit in a Rural Tertiary Care Hospital
}

\author{
Ravuri Usha Rani ${ }^{1}$, Bodduna Sameera ${ }^{2}$ \\ ${ }^{1}$ Department of General Medicine, PES Institute of Medical Sciences and Research, Kuppam, Andhra Pradesh, India. \\ ${ }^{2}$ Department of General Medicine, PES Institute of Medical Sciences and Research, Kuppam, Andhra Pradesh, India.
}

\section{ABSTRACT}

\section{BACKGROUND}

Hyponatremia is the most common electrolyte disorder in hospitalized patients. Its clinical diagnosis is difficult due to its marked variation in symptomatology as well as in its aetiology. As there are few studies on the clinical profile and aetiology of hyponatremia, the current study aims at evaluating the clinical and aetiological profile in patients admitted to a rural tertiary care hospital.

\section{METHODS}

It is a descriptive study in which patients more than 20 years of age who had serum sodium $<135 \mathrm{mEq} / \mathrm{L}$ within a study period of one year were included considering the inclusion and exclusion criteria. All relevant data were collected and analyzed.

\section{RESULTS}

Among the 70 study subjects, the mean age of distribution was $54.8 \pm 11.35$ years with male preponderance (63\%). Vomiting (35.7\%) was the most common complaint. Profound hyponatremia was seen in $37 \%$, whereas moderate hyponatremia in 33\%. Systemic hypertension (42.9\%) was the commonest comorbidity followed by diabetes mellitus (17.2\%), and diuretic use (57.1\%) was the commonest cause of hyponatremia followed by gastrointestinal (GI) losses $(21.3 \%)$. Hypovolemic hyponatremia was seen in $77 \%$ of the study subjects followed by hypervolemic hyponatremia in $17 \%$ and euvolemi bmb nc hyponatremia in $6 \%$.

\section{CONCLUSIONS}

As the aetiology of hyponatremia might be multifactorial, a meticulous approach is required for the correct diagnosis. As an accurate diagnosis can alter the outcome with timely intervention significantly reducing the high morbidity and mortality associated with the condition, a thorough understanding of its pathophysiology and the treatment strategy is essential.

\section{KEY WORDS}

Hyponatremia, Moderate, Profound, Hyponatremia, Diuretics, Hypovolemic Hyponatremia, Vomiting

\begin{abstract}
Corresponding Author:
Bodduna Sameera,

Assistant Professor,

Department of General Medicine,

PES Institute of Medical Sciences and

Research, Kuppam, Andhra Pradesh, India.

E-mail: sameera.bodduna@gmail.com
\end{abstract}

DOI: $10.14260 /$ jemds/2020/292

Financial or Other Competing Interests: None.

How to Cite This Article:

Rani RU, Sameera B. A clinical and aetiological study of hyponatremia in patients admitted to medical intensive care unit in a rural tertiary care hospital. J. Evolution Med. Dent. Sci. 2020;9(16): 1341-1344, DOI:
Submission 28-01-2020,

Peer Review 04-04-2020,

Acceptance 10-04-2020,

Published 20-04-2020. 


\section{BACKGROUND}

Electrolyte imbalances are one of the major complications seen in patients admitted to the medical intensive care unit (ICU). Hyponatremia is the most common electrolyte abnormality in hospitalized patients, $(1,2,3)$ and has also been associated with high mortality.(4) Hyponatremia is defined as serum sodium concentration $(\mathrm{Na}+)$ less than $135 \mathrm{mEq} / \mathrm{L} .{ }^{(5)}$ Homeostatic mechanisms like renal handling of the filtered sodium, anti-diuretic hormone, and thirst maintain the serum osmolality and serum sodium levels. Clinical symptoms may differ individually and can occur in a wide variety of patients who may be critically ill or sometimes even asymptomatic. Usually, patients are asymptomatic when their serum sodium levels are greater than $130 \mathrm{mEq} / \mathrm{L}$ and may show symptoms with much lower values. The majority of patients with hyponatremia have non-specific symptoms and may vary depending on the underlying disorder. As a result of comorbid conditions like cardiac, hepatic, and renal failure, elderly patients are more prone to hyponatremia, as observed in various studies.(6,7) Most of the hyponatremic clinical manifestations are due to cerebral edema, and the majority depends on the rate of fall of serum sodium levels rather than on its absolute level. Nausea, vomiting, and anorexia are the early symptoms of hyponatremia with headache and irritability in some patients. Neuropsychiatric symptoms occur when serum sodium levels fall even further; these range from simple restlessness, altered mental status, seizures to coma. Clinical diagnosis of hyponatremia is difficult due to marked variation in its symptomatology. The aetiology of hyponatremia varies from individual to individual, and so is the treatment strategy. The diagnostic and therapeutic approach to hyponatremia is multifactorial and it is classified into hypovolemic, hypervolemic and euvolemic hyponatremia depending on it. Attributing to the fact that it is multifactorial, an accurate history, clinical examination, and laboratory investigations are mandatory for the thorough evaluation of hyponatremia. The present study aims at assessing and evaluating the clinical profile, and aetiology of hyponatremia in patients admitted to medical ICU as early recognition and prompt treatment may reduce the symptom severity and mortality in hospitalized patients.

\section{METHODS}

This is a descriptive study conducted at PES (Peoples Educational Society) institute of medical sciences and research hospital (PESIMSR), Kuppam, Andhra Pradesh, South India, after obtaining the Hospital Ethical Committee clearance. All the patients who were admitted and had hyponatremia, who satisfied the inclusion criteria were selected, over a period of 12 months. This descriptive study was carried out on 70 consecutive subjects who admitted to PESIMSR medical ICU and are with serum sodium concentration $<135 \mathrm{mEq} / \mathrm{L}$ during the period from 2018 to 2019.

An elaborate history of events regarding onset, frequency and aetiological clues, past history as well as previous hospitalizations through old medical records were recorded. A complete general and systemic examination with detailed cardiovascular, respiratory, and neurological system examination was carried out on the patients. In addition to the routine laboratory investigations, chest radiograph (CXR) and electrocardiography (ECG) were done as and when required. The grading and severity of hyponatremia were done according to Joint European guidelines.(8) Patients were managed medically according to the underlying aetiology and those who required assisted ventilation like NIV; endotracheal intubation was managed in the intensive care unit (ICU).

\section{Inclusion Criteria}

- $\quad$ Patients aged more than 20 years of age.

- Patients with serum sodium < $135 \mathrm{mEq} / \mathrm{L}$.

All the cases were followed up until discharge or otherwise.

\section{Statistical Analysis}

All results are recorded, tabulated, and analyzed using appropriate statistical methods. The information collected regarding all the selected cases was recorded in a Master Chart. Data analysis was done with the help of a computer using MS-Excel, SPSS 22.0 (trial version).

\section{RESULTS}

The present study was conducted on 70 patients who were admitted to medical ICU with hyponatremia in PES medical college hospital, Kuppam, Chittoor, Andhra Pradesh.

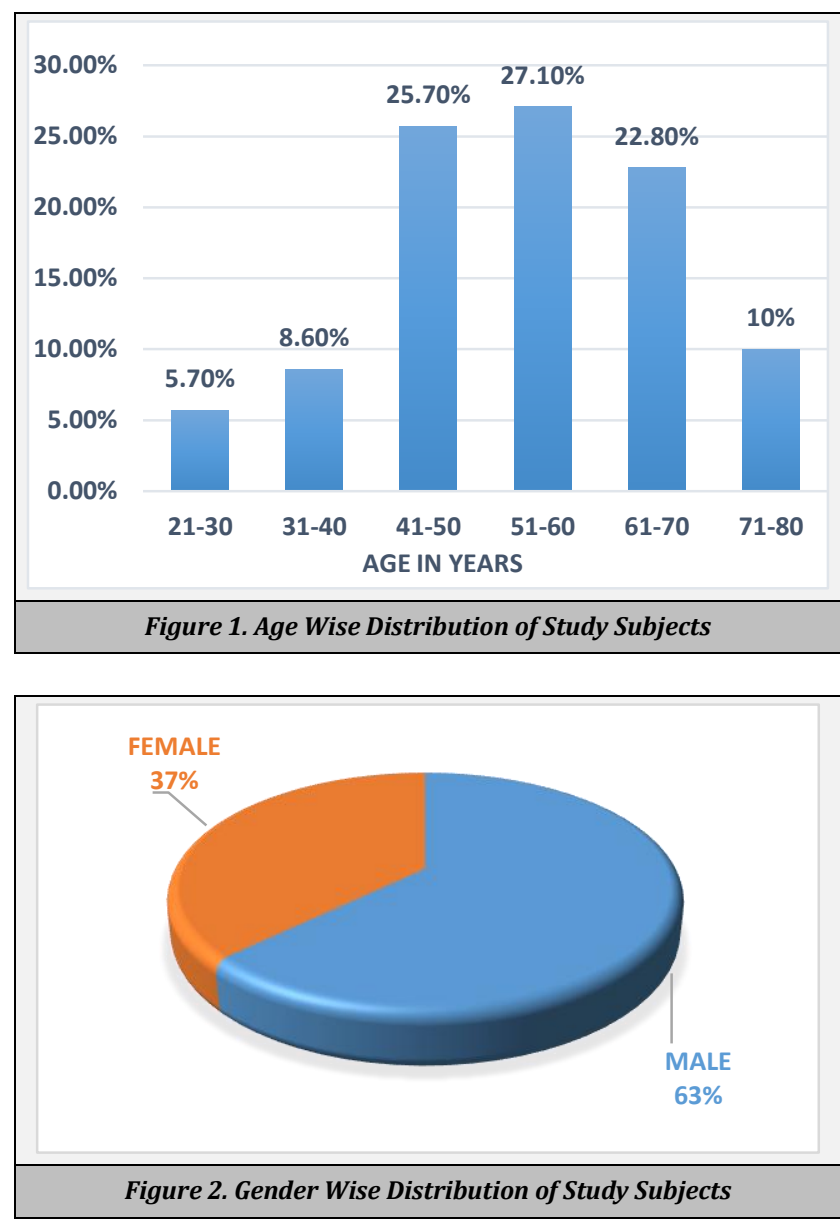


Among the total of 70 subjects, the maximum age group of distribution was between 51-60 years $(27.1 \%)$, the mean age being $54.8 \pm 11.35$ years, as shown in Fig. 1 . As shown in Fig. 2 , males were $63 \%$ and females $37 \%$ with a male: female ratio being 1.7:1. Among the 70 study subjects, the most common presenting complaint was vomiting (35.7\%) followed by CNS complaints [30\%-altered sensorium (10\%), drowsiness $(7.2 \%)$, giddiness (8.5\%), seizures (4.3\%)]. Among the 70 patients, hypertension as a comorbid condition was noted in $42.9 \%$, followed by diabetes mellitus in $17.2 \%$.

\begin{tabular}{|ccc|}
\hline Presenting Complaints & Patients & Percentage \% \\
Vomiting & 25 & 35.7 \\
CNS complaints & 21 & 30 \\
Breathlessness & 10 & 14.3 \\
Nausea & 7 & 10 \\
Diarrhoea & 7 & 10 \\
Total & $\mathbf{7 0}$ & $\mathbf{1 0 0}$ \\
\hline Table 1. Presenting Complaints among the Study Subjects \\
\hline
\end{tabular}
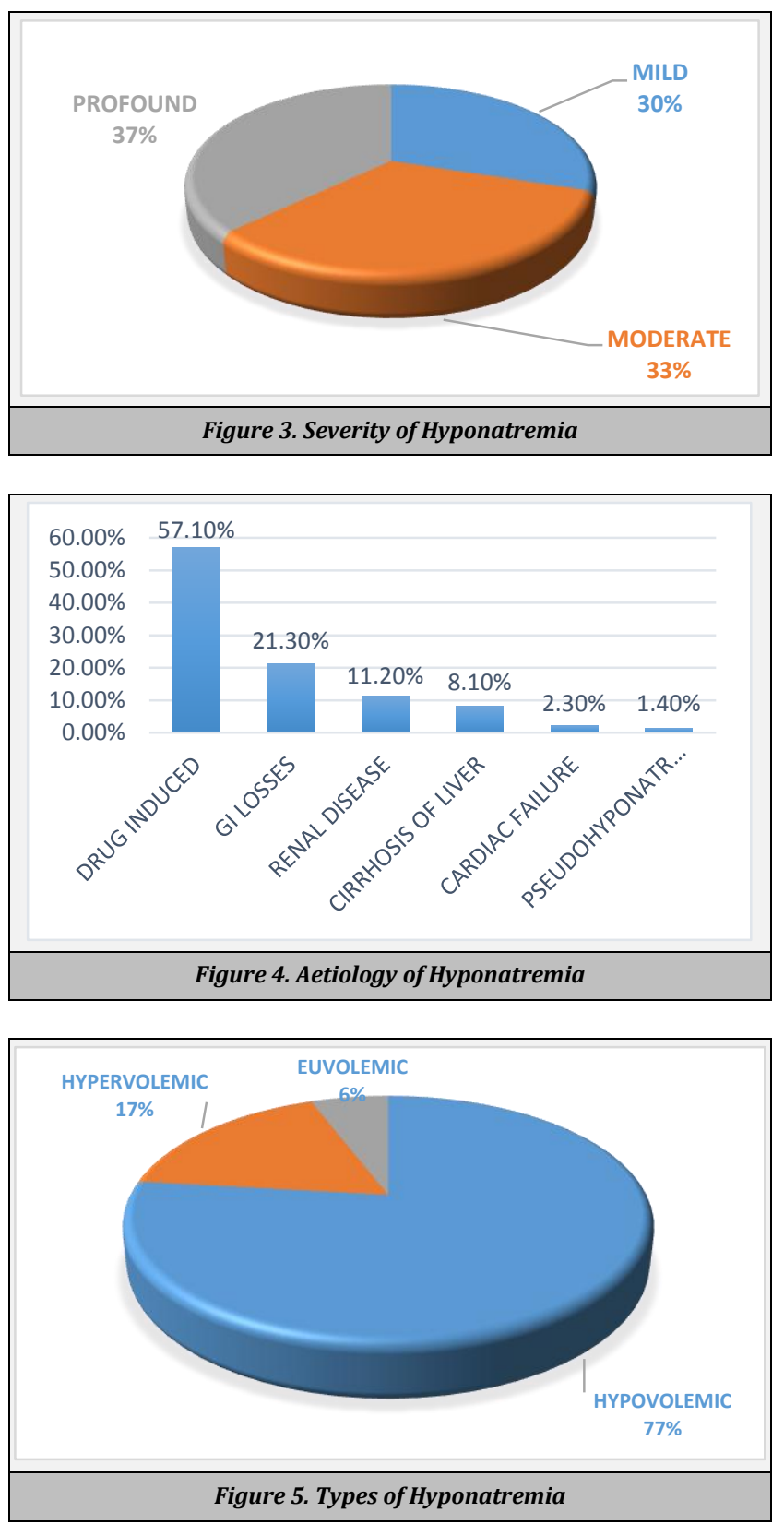

The serum sodium levels were $<125 \mathrm{mEq} / \mathrm{L}$ in $37 \%$ of the study subjects, whereas it was between $125-129 \mathrm{mEq} / \mathrm{L}$ in $33 \%$, as shown in fig. 3. As shown in fig. 4, hyponatremia was secondary to drug use in $57.1 \%$, followed by Gastrointestinal (GI) losses in $21.3 \%$. As shown in fig. 5 hypovolemic hyponatremia was seen in $77 \%$ of the study subjects followed by hypervolemic hyponatremia in $17 \%$ and euvolemic hyponatremia in $6 \%$.

\section{DISCUSSION}

Hyponatremia being the most common electrolyte imbalance, has a high significance in patients being admitted to ICU because of its multiple aetiologies and variable pathophysiological mechanisms. As the data on hyponatremia clinical profile and aetiology in ICU patients is scarce, the present study aims to discuss them both in patients admitted to the medical ICU in a tertiary health care center. In the present study, among 70 patients admitted with hyponatremia, $62.8 \%$ were males showing male preponderance. A similar pattern was reported by Bhattacharjee et al.(9) (72\% males), Baji.P. et al. (10) $(54 \%$ males) and Rahil et al.(11) (62.3\% males). The incidence of hyponatremia in our study was higher in the age group between $51-60$ years $(27.1 \%)$ with a mean age of 54.8 years. A similar distribution was reported by Bhattacharjee et al. (9) (58.6 years) and Baji.P. et al.(10) (59.4 years). As a result of impaired homeostasis of water and electrolytes, dietary variations, and comorbid conditions like cardiac, hepatic, and renal failure, the incidence of hyponatremia is much more in the elderly. The most common presenting complaint that was also considered as the commonest symptom in our study was vomiting (35.7\%) followed by CNS complaints (30\%) in comparison to study done by Maqbool et al.(12) where it was confusion (46\%), and Baji.P. et al. (10) reported nausea (54\%) as their commonest symptom. According to our study, the commonest comorbid condition was systemic hypertension (42.9\%), followed by diabetes mellitus (17.2\%). Similar results were reported by Bhattacharjee et al.(9) (57\%, 42\%) and Maqbool et al.(12) (51\%, 42\%). Diuretic use was shown to be a major and most common aetiological factor in the present study (57.1\%), as was also reported by Baji.P. et al. (10) (53\%). Among the 70 patients admitted, 37\% were profoundly hyponatraemic, followed by $33 \%$ with moderate hyponatremia in comparison to a study done by Rahil et al. (11), where profound hyponatremia was seen in $41.6 \%$ and moderate in $58.4 \%$. In the present study, the percentage of hypovolemic hyponatremia was 76\%, hypervolemic hyponatremia was $17 \%$, and euvolemic hyponatremia was seen in $6 \%$ of the admitted study patients. In comparison to the present study, in other studies reported hypovolemic hyponatremia in $47 \%,{ }^{(9)} 43 \%{ }^{(10)}$, and $46 \%{ }^{(12)}$ of study subjects. Hypervolemic hyponatremia was reported in $33 \%,{ }^{(9)} 38 \%,{ }^{(10)}$ and 37\%,(12) whereas euvolemic hyponatremia was seen in $20 \%,{ }^{(9)} 19 \%{ }^{(10)}$ and $17 \% .^{(12)}$

\section{CONCLUSIONS}

In ICU patients, hyponatremia is the most common electrolyte disorder. It is also a challenging disease due to its variable aetiology. As it might be multifactorial, it requires a meticulous approach to arrive at a correct diagnosis. As the 
identification of hyponatremia, its cause, along with the underlying systemic illness, carries a huge benefit in treating the disorder as well as in bringing down the morbidity and mortality associated, one should give utmost importance for the same. As an improper treatment towards hyponatremia can lead to serious neurological complications such as osmotic demyelination syndrome, every clinician should have knowledge regarding the approach and treatment strategy for hyponatremia.

\section{REFERENCES}

[1] Gonc NE, Kandemir N, Sen Y, et al. Hyponatremia can be a presenting finding of multiple pituitary hormone deficiency in children: report of a case and review of literature. Clin Pediatr (Phila) 2005;44(7):623-8.

[2] Bhananker SM, Paek R, Vavilala MS. Water intoxication and symptomatic hyponatremia after outpatient surgery. Anesth Analg 2004;98(5):1294-6.

[3] Coenraad MJ, Meinders AE, Vandenbroucke JP, et al. Causes of hyponatremia in the departments of internal medicine and neurosurgery. European Journal of Internal Medicine 2003;14(5):302-9.

[4] Douglas I. Hyponatremia: Why it matters, how it presents, how we can manage it? Cleveland Clinic Journal of Medicine 2006;73(Suppl 3):S4-S12.
[5] Hoorn EJ, Zietse R. Diagnosis and treatment of hyponatremia: compilation of the guidelines. J Am Soc Nephrol 2017;28(5):1340-9.

[6] Fegan G, Begley J. Hyponatremia in the elderly. CME Geriatr Med 2005;7:76-85.

[7] Schrier RW. Body fluid volume regulation in health and disease: a unifying hypothesis. Ann Intern Med 1990;113(2):155-9.

[8] Spasovski G, Vanholder R, Allolio B, et al. Clinical practice guideline on diagnosis and treatment of hyponatraemia. Nephrol Dial Transplant 2014;(29 Suppl 2):i1-i39.

[9] Bhattacharjee P, Das P, Das D, et al. Clinical and aetiological profile of patients presenting with hyponatremia in a tertiary care teaching hospital of North Eastern India. International Journal of Contemporary Medical Research 2017;4(5):1038-41.

[10] Baji PP, Borkar SS. Clinico-etiological profile and outcome of hyponatremia in hospitalised adult patients. Int J Sci Rep 2015;1(7):293-8.

[11] Rahil AI, Khan FY. Clinical profile of hyponatraemia in adult patients admitted to Hamad General Hospital, Qatar: experience with 53 cases. J Clin Diag Res 2009;3:1419-25.

[12] Maqbool M, Raina AA, Wani BA, et al. Symptomatic hyponatremia, aetiology and outcome in a tertiary care hospital. Int J Adv Res 2016;4(12):1781-4. 